\title{
Rotation in molecular clouds ${ }^{\star}$
}

\section{J.P. Phillips}

Instituto de Astronomía y Meteorología, Universidad de Guadalajara, Av. Vallarta 2602, CP. 44100 Sector Juárez, Guadalajara, Jalisco, Mexico

e-mail: jpp@udgserv.cencar.udg.mx

Received February 23; accepted July 31, 1998

\begin{abstract}
We have investigated a broad range of evidence concerning rotation in molecular clouds. As a consequence, we show that trends in specific angular momentum $J / M$ and angular velocity $\Omega$ are inconsistent with certain models of isothermal, non-magnetic cloud rotation. Similarly, models of rotation which invoke turbulent vorticity may have only limited applicability to clumps and condensations. There is evidence to favour an important rôle for rotation in maintaining the stability of disks, larger cloud structures, and perhaps a large fraction of intermediate sized clouds, whilst rotation may also be implicated in maintaining observed departures from cloud sphericity. Although it is conceivable that magnetic braking is responsible for the radial decrement in specific angular momentum, it appears that observed gradients $\operatorname{dln}(J / M) / \operatorname{dln}(R)$ are significantly shallower than is normally anticipated through this mechanism.
\end{abstract}

The variation of angular momentum with cloud mass $M$ (viz. $J \propto M^{1.7}$ ) appears to be highly correlated, and is consistent with models of clump merging in isothermal rotating clouds. Similarly, the orientations of the angular velocity vectors for clumps and condensations appear broadly random, suggesting a turbulent origin for observed components of $\Omega$ or, alternatively, a process of randomisation through magnetic and/or dynamic clump interactions. By contrast, isolated clouds (and perhaps also disks) are shown to have angular velocity vectors oriented predominantly towards the north and south galactic poles; a distribution which would be anticipated were components of $J$ to arise from galactic shear.

We show, finally, that most of the cloud subgroups appear to follow similar functional trends in $J, J / M$, and $\Omega$. Disks and rings, on the other hand, appear to depart from these variations to a significant degree; a difference which

Send offprint requests to: J.P. Phillips

* Table 1 is only available at the CDS in electronic from via ftp to cdsarc.u-strasbg.fr (130.79.128.5) or via http://cdsweb.u-strasbg.fr/Abstract.html presumably derives from their distinct spatio-kinematic structures.

Key words: ISM: clouds - ISM: kinematics and dynamics — ISM: molecules — ISM: structure

\section{Introduction}

Observations of molecular clouds at millimetric wavelengths suggest that these structures are prone to a broad variety of kinematic disturbances. In particular, and at the largest scales, there appears to be frequent evidence for systematic gradients in velocity, extending (in certain cases) over tens of parsecs. Such gradients may arise through a broad variety of mechanisms, and it seems likely that dynamical shearing between clouds and the ambient medium (e.g. Arquilla \& Goldsmith 1986; Goldsmith \& Sernyak 1984; McCutcheon et al. 1986), chance superposition of kinematically differing cloud structures (Thronson et al. 1985; Wang et al. 1993; Ziurys et al. 1981), outflows from young stars (e.g. Tafalla et al. 1993), and the collective impact of winds emerging from HII regions, OB associations, supernovae and so forth (e.g. Gonzalez-Alfonso et al. 1995; Patel et al. 1993; Blitz 1993) may lead to similar variations.

In the large majority of cases, however, such systematic trends in LSR velocity have been attributed to cloud rotation.

The importance of rotation in the evolution of molecular clouds is far from adequately established. Whilst certain upper limit estimates for velocity gradient have been used to adduce a comparatively small contribution to cloud stability (Myers et al. 1991; Nozawa et al. 1991; Myers \& Benson 1983; Loren et al. 1983; Dickman \& Clemens 1983), other observations suggest that rotation may play a formative and important role in at least certain clouds, complexes (e.g. Field 1978; Blitz 1993; Arquilla \& 
Goldsmith 1985, 1986), and disks (e.g. Jackson et al. 1988; Vogel et al. 1985).

The origins of such rotation remain uncertain, as is the importance of this contribution at various stages of cloud evolution. Thus, and most commonly, it is envisaged that there is a transfer of angular momentum from large to small scales as complexes contract and fragment, with radiation of Alfven waves and inter-clump interactions subsequently leading to various degrees of rotational braking. By contrast, Fleck \& Clark (1981) have suggested that rotation in clouds may derive from the vorticity associated with IS turbulence.

These and other mechanisms imply differing distributions of angular momentum with cloud radii, and have implications for the comparative importance of angular momentum in maintaining cloud stability, and the orientations of angular momentum vectors with respect to the galactic plane.

Although there have been several previous attempts to assess the properties of cloud angular momentum from observations, systematic studies of this phenomenon have been restricted to comparatively few sources (e.g. Fleck \& Clark 1981; Field 1978; Goldsmith \& Arquilla 1985; Arquilla \& Goldsmith 1986; Heyer 1988; Casali \& Edgar 1987), and have come (in certain cases) to indecisive or contradictory conclusions.

In the following, we compile a data base of cloud rotational measures which is at least an order of magnitude greater than has been investigated heretofore. This is used to evaluate overall trends in angular momentum, the orientation of angular momentum vectors, the stability of molecular clouds, and the role of rotation in determining departures from cloud sphericity. We shall find that most current models for the evolution of rotating clouds are to varying degrees inadequate.

\section{Cloud rotational observations}

The data summarised in Table 1 has been compiled from published sources dating between 1972 and the present. It includes a broad range of information for clouds where velocity gradients have been attributed to rotation. In a few cases, explanations for these gradients have additionally been sought in terms of other mechanisms, and certain of these suggestions are indicated in the footnotes. In particular (and as noted in Sect. 1), the superposition of kinematically separate components (see discussion in Sect. 4), or tilted aspherical outflows may lead to very similar projected kinematic structures; it is often extremely difficult to distinguish between these various mechanisms.

For the large majority of sources in the present sample, however, rotation has been regarded as the most likely and sole explanation for observed large scale velocity gradients.

Certain of the clouds have been observed several times, although for these cases we have included only a representative sample of observations in Table 1. As a consequence, the table includes data referring to 111 individual sets of observations, and 156 differing sources and/or spatially distinct regions within the same source.

The various data headings may be summarised as follows. Columns 1-7 refer to the cloud designation, coordinates, distance, LSR velocity, and line width as detailed in the various references cited in Col. 15; in a very few cases, we have altered quoted distances where previous estimates conflict with more recent values. Similarly, we have (where possible) used line widths corresponding to low optical depth transitions. The dimension $L$ (Col. 7 ) refers to the projected region of cloud over which gradients were measured. Column 8 indicates the molecular transitions used in acquiring the results, whilst $\Omega$ corresponds to the angular rotational velocity deduced from projected velocity gradients. $\Gamma$ is an estimate of cloud axial ratio (major axis/minor axis) based on the spatial variation in emission contours over the projected region of rotation, whilst $M$ is the non-virial mass of the zone based (usually) on estimates of column density (values $M_{\text {ast }}$ (in parentheses) correspond to the embedded stellar mass). $\Theta$ (Col. 12) represents the projected orientation of the angular velocity vector $\Omega$ with respect to the galactic plane, whereby a vector $\Omega$ oriented towards the north galactic pole is taken to have an inclination $\Theta=90^{\circ}$, whilst one pointing towards the south galactic pole has $\Theta=270^{\circ}$. The orientation of this vector relative to the cloud major axis is also represented (Col. 13), whereby we indicate those clouds for which $\Omega$ resides within $30^{\circ}$ of the minor axis (designated $Y$; that is, the maximum velocity gradient lies along the cloud major axis), and those cases where this is not the case (designated $N$ ).

Finally, we have attempted to broadly categorise the regions based largely on descriptions provided in the references. Thus, MS, MI, and ML are, respectively, isolated small, intermediate, and large clouds (this latter category also including, and largely consisting of complexes). Condensations within such regions are adjectivaly indicated through the appended letter $\mathrm{C}$ (thus MIC is a condensation within an intermediate sized cloud), whilst filamentary cloud structures are represented by F. Finally, clumps are designated by CL, whilst rings and disks are indicated by $D / R$.

Note that in order to represent this data on a uniform basis, it has often been necessary to evaluate parameters using published maps and spectra, rather than employing values quoted in the respective references.

Finally, the results of a least squares analysis for selective logarithmic rotational parameters is summarised in Table 2, where $F(y)=a+b F(x)$, and we include determinations for angular momentum $J$, specific angular momentum $J / M$, non-virial mass $M$, and angular velocity $\Omega . N$ is the total sample number, and $r$ is the correlation coefficient. The nature of these trends will be discussed more fully in the proceeding sections. 
Table 2. Parametric trends in molecular clouds

\begin{tabular}{|c|c|c|c|c|c|c|}
\hline$F(y)$ & $F(x)$ & Cloud Set & $N$ & $a$ & $b$ & $r$ \\
\hline $\log \left(\Omega /\left(10^{14} s\right)\right)$ & $\log (R / \mathrm{pc})$ & ALL & 163 & 0.35 & -0.73 & 0.71 \\
\hline $\log \left(\Omega /\left(10^{14} \mathrm{~s}\right)\right)$ & $\log (R / \mathrm{pc})$ & CLOUDS & 63 & 0.36 & -0.53 & 0.67 \\
\hline $\log \left(\Omega /\left(10^{14} \mathrm{~s}\right)\right)$ & $\log (R / \mathrm{pc})$ & CONDS. & 69 & 0.31 & -0.60 & 0.51 \\
\hline $\log \left(\Omega /\left(10^{14} s\right)\right)$ & $\log (R / \mathrm{pc})$ & $\mathrm{D} / \mathrm{R}$ & 31 & 0.31 & -1.04 & 0.84 \\
\hline $\log \left(\Omega /\left(10^{14} \mathrm{~s}\right)\right)$ & $\log \left(M / M_{\odot}\right)$ & ALL & 112 & 0.83 & -0.11 & 0.20 \\
\hline $\log \left(\Omega /\left(10^{14} s\right)\right)$ & $\log \left(M / M_{\odot}\right)$ & CLOUDS & 38 & 0.70 & -0.11 & 0.25 \\
\hline $\log \left(\Omega /\left(10^{14} \mathrm{~s}\right)\right)$ & $\log \left(M / M_{\odot}\right)$ & CONDS. & 70 & 0.51 & -0.01 & 0.03 \\
\hline $\log \left(\Omega /\left(10^{14} \mathrm{~s}\right)\right)$ & $\log \left(M / M_{\odot}\right)$ & $\mathrm{D} / \mathrm{R}$ & 24 & 1.65 & -0.30 & 0.41 \\
\hline $\log (J / M(\mathrm{~km} / \mathrm{s} / \mathrm{pc}))$ & $\log \left(M / M_{\odot}\right)$ & ALL & 112 & -2.42 & 0.72 & 0.88 \\
\hline $\log (J / M(\mathrm{~km} / \mathrm{s} / \mathrm{pc}))$ & $\log \left(M / M_{\odot}\right)$ & CLOUDS & 38 & -2.29 & 0.79 & 0.92 \\
\hline $\log (J / M(\mathrm{~km} / \mathrm{s} / \mathrm{pc}))$ & $\log \left(M / M_{\odot}\right)$ & CONDS. & 50 & -2.42 & 0.64 & 0.88 \\
\hline $\log (J / M(\mathrm{~km} / \mathrm{s} / \mathrm{pc}))$ & $\log \left(M / M_{\odot}\right)$ & $\mathrm{D} / \mathrm{R}$ & 24 & -2.49 & 0.66 & 0.87 \\
\hline $\log (J / M(\mathrm{~km} / \mathrm{s} / \mathrm{pc}))$ & $\log (R / \mathrm{pc})$ & ALL & 163 & -0.56 & 1.27 & 0.87 \\
\hline $\log (J)$ & $\log (R)$ & CLOUDS & 63 & -0.55 & 1.47 & 0.93 \\
\hline $\log (J / M(\mathrm{~km} / \mathrm{s} / \mathrm{pc}))$ & $\log (R / \mathrm{pc})$ & CONDS. & 69 & -0.59 & 1.40 & 0.81 \\
\hline $\log (J / M(\mathrm{~km} / \mathrm{s} / \mathrm{pc}))$ & $\log (R / \mathrm{pc})$ & $\mathrm{D} / \mathrm{R}$ & 31 & -0.60 & 0.96 & 0.82 \\
\hline $\log \left(J\left(M_{\odot} \mathrm{km} / \mathrm{s} / \mathrm{pc}\right)\right)$ & $\log \left(M / M_{\odot}\right)$ & ALL & 112 & -2.42 & 1.72 & 0.98 \\
\hline $\log \left(J\left(M_{\odot} \mathrm{km} / \mathrm{s} / \mathrm{pc}\right)\right)$ & $\log \left(M / M_{\odot}\right)$ & CLOUDS & 38 & -2.01 & 1.73 & 0.96 \\
\hline $\log \left(J\left(M_{\odot} \mathrm{km} / \mathrm{s} / \mathrm{pc}\right)\right)$ & $\log \left(M / M_{\odot}\right)$ & CONDS. & 50 & -2.42 & 1.64 & 0.98 \\
\hline $\log \left(J\left(M_{\odot} \mathrm{km} / \mathrm{s} / \mathrm{pc}\right)\right)$ & $\log \left(M / M_{\odot}\right)$ & $\mathrm{D} / \mathrm{R}$ & 24 & -2.49 & 1.66 & 0.97 \\
\hline $\log \left(J\left(M_{\odot} \mathrm{km} / \mathrm{s} / \mathrm{pc}\right)\right)$ & $\log (R / \mathrm{pc})$ & ALL & 112 & 1.74 & 2.75 & 0.86 \\
\hline $\log \left(J\left(M_{\odot} \mathrm{km} / \mathrm{s} / \mathrm{pc}\right)\right)$ & $\log (R / \mathrm{pc})$ & CLOUDS & 38 & 1.50 & 3.28 & 0.94 \\
\hline $\log \left(J\left(M_{\odot} \mathrm{km} / \mathrm{s} / \mathrm{pc}\right)\right)$ & $\log (R / \mathrm{pc})$ & CONDS. & 70 & 1.93 & 3.34 & 0.83 \\
\hline $\log \left(J\left(M_{\odot} \mathrm{km} / \mathrm{s} / \mathrm{pc}\right)\right)$ & $\log (R / \mathrm{pc})$ & $\mathrm{D} / \mathrm{R}$ & 24 & 1.75 & 2.56 & 0.88 \\
\hline $\log \left(M / M_{\odot}\right)$ & $\log (R / \mathrm{pc})$ & ALL & 109 & 2.37 & 1.45 & 0.78 \\
\hline $\log \left(M / M_{\odot}\right)$ & $\log (R / \mathrm{pc})$ & CLOUDS & 38 & 2.16 & 1.76 & 0.89 \\
\hline $\log \left(M / M_{\odot}\right)$ & $\log (R / \mathrm{pc})$ & CONDS. & 47 & 2.54 & 1.82 & 0.76 \\
\hline $\log \left(M / M_{\odot}\right)$ & $\log (R / \mathrm{pc})$ & $\mathrm{D} / \mathrm{R}$ & 24 & 2.52 & 1.19 & 0.75 \\
\hline
\end{tabular}

\section{Cloud rotational properties}

The information cited in Table 1 may be used to investigate a broad range of cloud rotational properties. In the following, we separately investigate variations in specific and total angular momentum, cloud orientation, angular velocity, stability and morphology, comparing the results with trends anticipated from theoretical analyses.

\subsection{Orientations}

Where cloud angular momentum derives from shear in the galactic disk, and there is no subsequent dynamical or magnetic interaction with other entities, then one would expect to find strong residual evidence for preferential orientation of the angular momentum vectors. In particular, the orientation of $\Omega$ depends upon the relative values of the Oort constants A and B, and the initial intrinsic cloud aspect ratio $\Gamma_{\mathrm{i}}$ (e.g. Field 1978); although in general, one would anticipate values $\Theta=90^{\circ}$ or $270^{\circ}$. Where, on the other hand, such rotation arises through turbulence, or is alternatively strongly disordered through (say) magnetic coupling between clumps, then one might expect that the orientations in $\Omega$ would be largely random.

What, from the present evidence, can one say regarding such trends in molecular clouds?
The data is represented in the form of a series of histograms in Figs. 1a-c. It is clear, from these, that if we consider only the individual clouds (that is, we eliminate clumps, condensations, disks or rings), then there is a reasonably strong indication of preferential orientation, with approximately equal numbers of vectors $\Omega$ oriented towards the north and south galactic poles. The application of the Kolmogorov-Smirnov test to such a distribution yields a probability which depends, partially, upon which point in the angular sequence one chooses to commence with. If the lowest point in the data sequence is taken to be $\theta=250^{\circ}$, then the probability of the doublepeaked structure arising by chance is quite small, of order $2.810^{-2}$. If, on the other hand, the data sequence is folded about $\theta=180^{\circ}$, so that the peaks approximately coincide, then the probability reduces to $\sim 510^{-10}$. Whatever statistical test one might choose to employ, therefore, it is likely that the probability of Fig. 1a arising by chance is going to be quite small.

Finally, we note that disks and rings also appear to share some evidence for preferential orientation (Fig. 1b), although clumps and condensations (which might be expected to possess a similar evolutionary pedigree) have a distribution which is essentially random (Fig. 1c). 

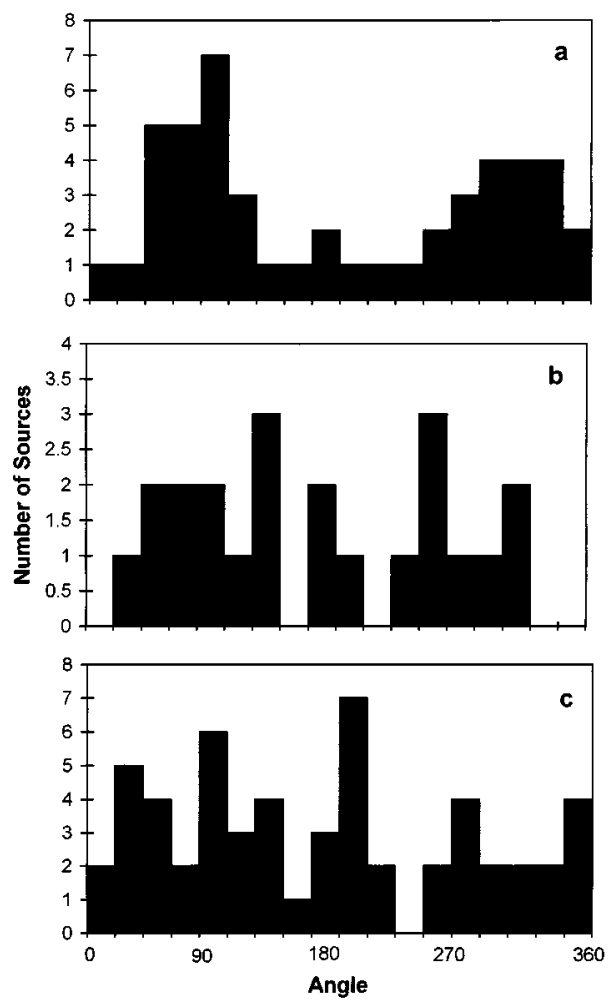

Fig. 1. a) Distribution of molecular clouds as a function of angle $\Theta$ between the angular velocity vector $\Omega$ and galactic plane, where we have illustrated the subgroup of isolated clouds (i.e. cloud types MI, MC, etc.). b) As for Fig. 1a, but for disks and rings (i.e. subgroup $D / R$ ). c) As for Fig. 1a, but for clumps and condensations

Such trends are sufficiently disparate to confound any attempt at a simple explanation. Clouds, for instance, may acquire their momenta through shearing motions in the galactic disk, as noted above. Angular momentum in clumps, by contrast, is unlikely to derive through spin-up of gravitationally unstable sub- regions within larger cloud systems; or alternatively, if such spin-up did in the past occur, then the vector direction may have been randomised through dynamical or magnetic interactions. Given such a situation, it is then however unclear why disks may have retained some fossil memory of their original orientations one would anticipate that these, too, should show random orientations.

\subsection{Variations in angular velocity}

The variation of angular velocity with cloud radius $(\equiv L / 2)$ is represented in Fig. 2 for all of the cloud sample, where solid lines connect results determined for differing regimes of the same (differentially rotating) disks. A least squares analysis of these trends (Table 2) suggests that clouds and condensations taken separately are characterised by gradients $\operatorname{dn} \Omega / \operatorname{dln} R \cong-0.59$; a value which is similar to that determined for the sample as a

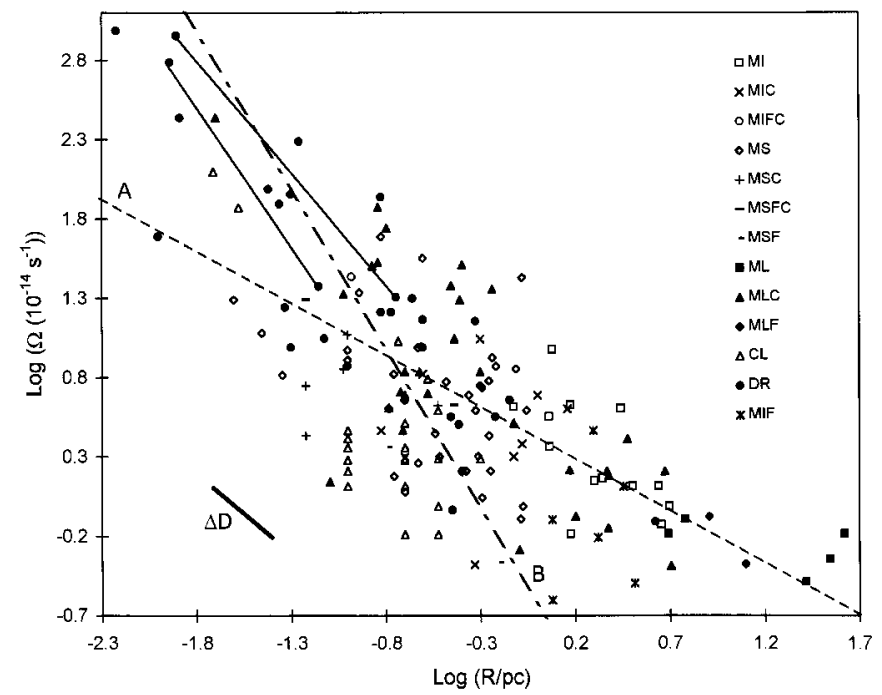

Fig. 2. Variation of projected cloud angular velocity $\Omega$ with radius for all of the clouds in the present study; the right hand key identifies various subgroups (see text for details). Solid lines connect régimes within differentially rotating disks, whilst the dashed lines represent expected trends for turbulent vorticity (A) and angular momentum conservation (B). Finally, the broad diagonal line (designated $\Delta D$ ) corresponds to the variation in source position resulting from a factor 2 change in distance

whole. The disks and rings, on the other hand, display the tightest correlation between $\Omega$ and $R$ (the correlation coefficient is $r=0.84$ ), and are characterised by a gradient $\operatorname{d} \ln \Omega / \operatorname{dln} R \cong-1.04$ which is considerably steeper.

We have also included in Fig. 2 the trends which would be anticipated were angular momentum conserved, and those predicted through the turbulent vorticity model of Fleck \& Clark (1981). Neither represents observed trends particularly well. Thus, the observed variation of $\Omega$ with $R$ is significantly less steep than would be envisaged were angular momentum conserved - a disparity which might be regarded as favouring models in which $J$ is leached through a process of magnetic braking. If this were the case, then it must be concluded that such a mechanism must operate over all cloud sizes $R \leq 4 \mathrm{pc}$. Whilst the variation in $\Omega$ for larger cloud sizes is rather less clear, it appears on current evidence to be relatively modest.

The variation of $\Omega$ with mass is particularly interesting, since the correlation between these two parameters appears in all cases to be very low (Fig. 3); indeed, it would appear that the relevant figures constitute a species of scatter diagram, with correlation coefficients ranging between 0.03 and 0.41 (the latter value corresponding to the disks; the only subgroup to display evidence for systematic trends (Table 2)). As we shall see later, this lack of correlation is in marked contrast to the corresponding variation in angular momentum $J(M)$.

Finally, two final points are worth making. Although the contribution of uncertainties in distance $D$ is 


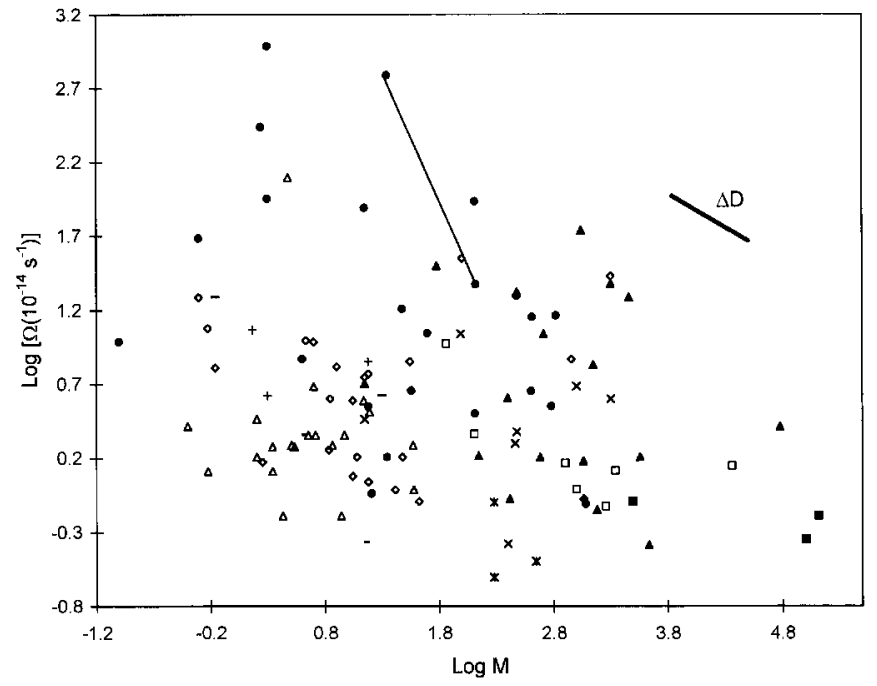

Fig. 3. Variation of angular velocity $\Omega$ with cloud mass $M$ (where both here and the proceeding figures, $M$ is given in units of the solar mass)

difficult to quantify precisely, it seems unlikely that estimates can be in error by greater than a factor 2 . Thus, whilst certain of the larger values $D$ may be open to appreciable revision, the mean influence of such uncertainties is likely to be modest. This is illustrated in Figs. 2 and 3 by means of broad diagonal bars (labelled $\Delta D$ ) wherein is indicated the variation in cloud location arising from a factor 2 change in distance. Similar loci are illustrated in the remaining figures. This question is also further analysed in the appendix, where we conclude (from an analysis of $10^{3}$ model clouds) that even order of magnitude errors in distance would be unlikely to reproduce observed gradients and correlation coefficients.

The second point concerns the role of cloud projection in modifying deduced rotational properties. In particular, where the angular velocity vector $\Omega$ is inclined at some angle $>0^{\circ}$ to the plane of the sky, then intrinsic angular momentum $J$, angular velocity $\Omega$, and a raft of other parameters (including $J / M$ (Sect. 3.3), $\alpha$ and $\beta$ (Sect. 3.4) and $\Gamma$ (Sect. 3.5)) are likely to be undervalued. Similarly, since the projected mean values of such parameters depend, in part, upon cloud morphology, then any systematic trend of morphology with radius may lead to changes in the gradients $\operatorname{dn} J / \operatorname{dn} R, \operatorname{dln} \Omega / \operatorname{dln} R$ and so forth. Such a possibility must in fact be taken quite seriously when considering the clouds as a whole, since many larger clouds appear to be spindle shaped, whilst disks are concentrated towards the lower end of the radial range.

Although such effects may be far from negligible, they are also extremely difficult to quantify precisely. Taken as a whole, however, it seems unlikely that the (statistical mean) trends and conclusions of this analysis would be greatly modified by such corrections.

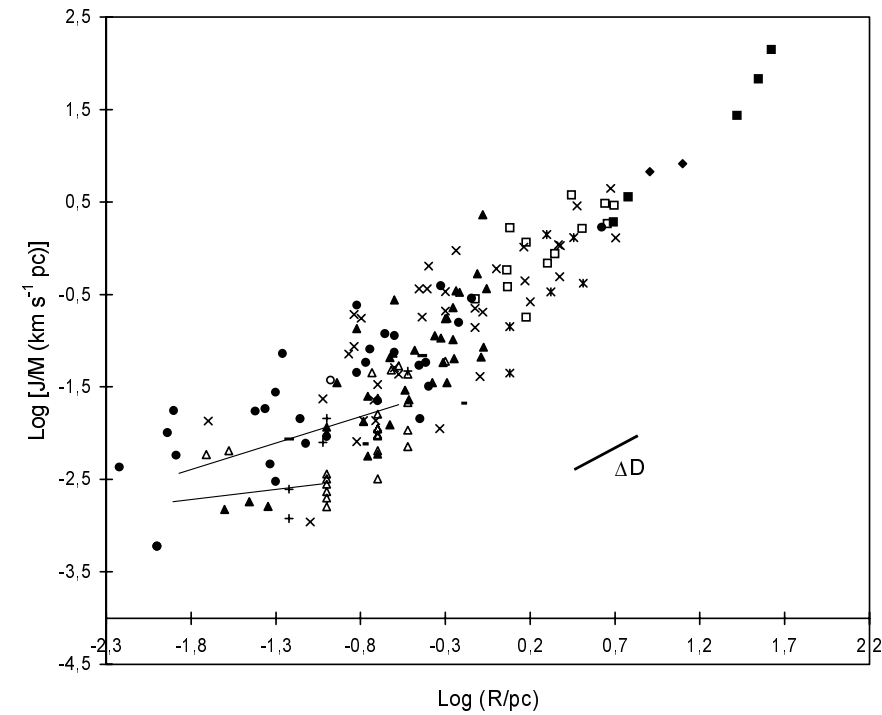

Fig. 4. Variation of specific angular momentum $J / M$ as a function of cloud radius $R$, where we have included all of the cloud subgroups in the present study (see Fig. 2 for key to symbols)

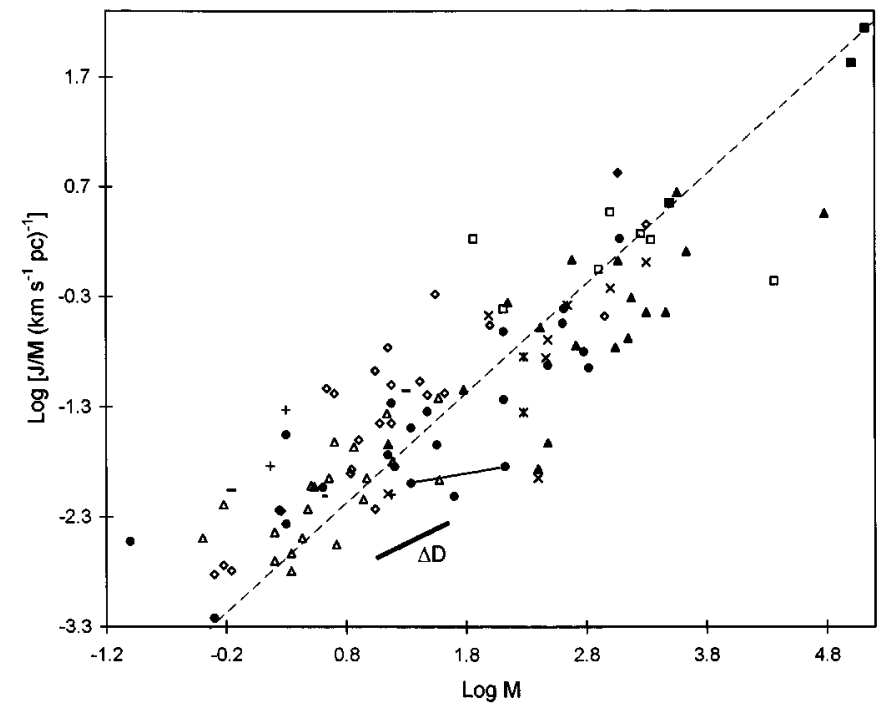

Fig. 5. As in Fig. 3, but for the variation of $J / M$ with mass $M$. The diagonal line corresponds to the trend expected for isothermal, non-magnetic rotating clouds

\subsection{Specific and total angular momentum}

The variation of specific angular momentum with radius is illustrated for the complete data set in Fig. 4, where $J / M \equiv \phi \Omega R^{2}$, and the parameter $\phi$ varies from 0.5 in the case of disks through to 0.33 for prolate structures; we shall adopt an intermediate value $\phi=0.4$ appropriate for spheres. It is clear, from this, that $J / M$ declines systematically with decreasing cloud radius, and that the gradient is of order $\operatorname{dln}(J / M) / \operatorname{dln} R \cong 1.43$ for both isolated clouds and condensations. On the other hand, disks again display a trend which is disparate from that of other regions in this study, yielding a gradient $\operatorname{d} \ln (J / M) / \operatorname{dnn} R \cong 0.96$. 


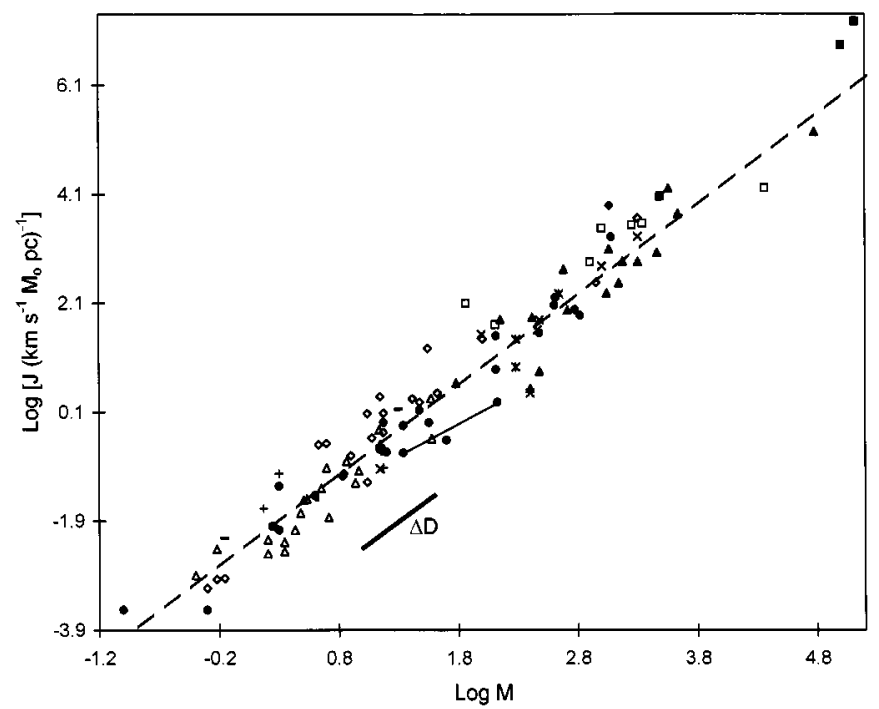

Fig. 6. Variation of angular momentum $J$ with cloud mass $M$. The diagonal line represents the trend anticipated for simple clump merger models (Benz 1984)

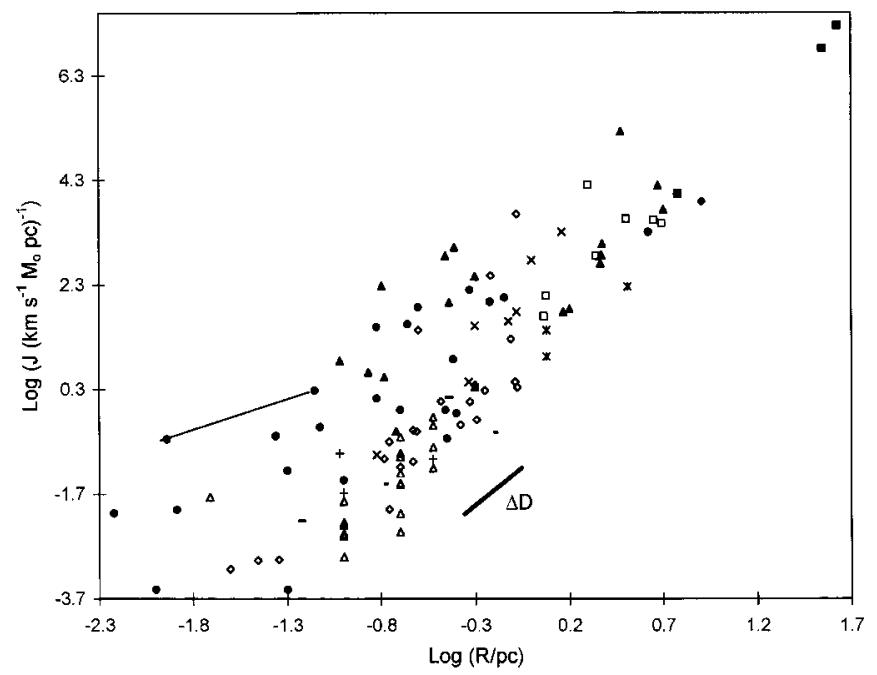

Fig. 7. Variation of angular momentum $J$ with cloud radius $R$

This difference largely disappears when we consider the variation of $J / M$ with mass $M$, on the other hand (Fig. 5; Table 2), whence it is clear that all of the cloud components share comparable gradients $\operatorname{dln}(J / M) / \operatorname{d} \ln M \cong 0.7$. It is interesting, in this case, to note that models of isothermal, non-magnetic rotating clouds would require $J / M$ to scale as $M$ (i.e. $\operatorname{dln}(J / M) / \operatorname{dln} R$ takes a value unity; Bodenheimer \& Black 1978). Indeed Boss (1987), in his discussion of model rotating disks, concludes that some such scaling law is consistent with observed trends in disklike structures.

The gradient indicated by the present observations appears, in reality, to be somewhat shallower, suggesting that such analyses may be inapplicable to real cloud structures.
The variation of angular momentum $J$ with mass (Fig. 6) constitutes one of the tightest correlations in the present data set $(r \cong 0.98)$; a feature which is all the more interesting given that $J$ depends upon a total of three independent parameters $(R, M \& \Omega)$ and their associated errors. Although the scale range in $J$ is rather larger than that of other functions investigated here - and this, in turn, can lead to an apparent enhancement in correlation - we shall find below that this is by no means the entire story.

As might be expected from the narrowness of the distribution in Fig. 6, clumps, disks and clouds share closely similar gradients $\operatorname{d} \ln J / \operatorname{d} \ln M \cong 1.7$; a value which is closely similar the model gradient $\operatorname{dln} J / \operatorname{dln} M \cong 5 / 3$ noted for clumps in non-magnetic rotating clouds (Benz 1984).

If one now determines the variation of $J$ with $R$, as in Fig. 7, we obtain a distribution which is qualitatively somewhat different; the scatter in results appears to be broader, and correlation coefficients $(r \sim 0.88)$ are correspondingly lower. Most of the gradients for individual cloud sub-groups are also rather similar (of or$\operatorname{der} \operatorname{dln} J / \operatorname{dln} R \sim 3.3$ ), although it appears that disks are again different in having a gradient some $30 \%$ smaller.

It follows, from this, that the decrease in angular momentum appears to be directly related to the decrease in mass, and less so to changes in radius; it is difficult to avoid the conclusion that these various distributions are telling us something rather important about the way angular momentum is transferred from large to small scales. At the very least, one might infer that rotational braking processes must be strongly mass dependent, whilst rotational mechanisms which depend primarily upon size scale (cf. turbulent vorticity) would be unlikely to reproduce the very tight correlations noted above. Similarly, we note that where cloud angular velocities derive from galactic shear, then the value of $|J|$ will depend upon the morphology and orientation of the initial cloud structure; it is possible for $J$ to possess a comparatively broad range of values for any single cloud mass $M$ (see, for instance, the discussion in Blitz 1993). Under these circumstances, it follows that the tight correlation noted in Fig. 6 would imply closely similar initial collapse configurations.

\subsection{Cloud stability}

There are various means whereby one might investigate the importance of rotation for overall cloud stability. In particular, it is possible (for instance) to define a ratio $\beta$ between the rotational and combined turbulent and thermal virial terms through

$\beta=7.0410^{-2}\left[\frac{R}{\mathrm{pc}}\right]^{2}\left[\frac{\Omega}{10^{-14} \mathrm{~s}}\right]^{2}\left[\frac{\Delta V}{\mathrm{~km} \mathrm{~s}^{-1}}\right]^{-2}$ 


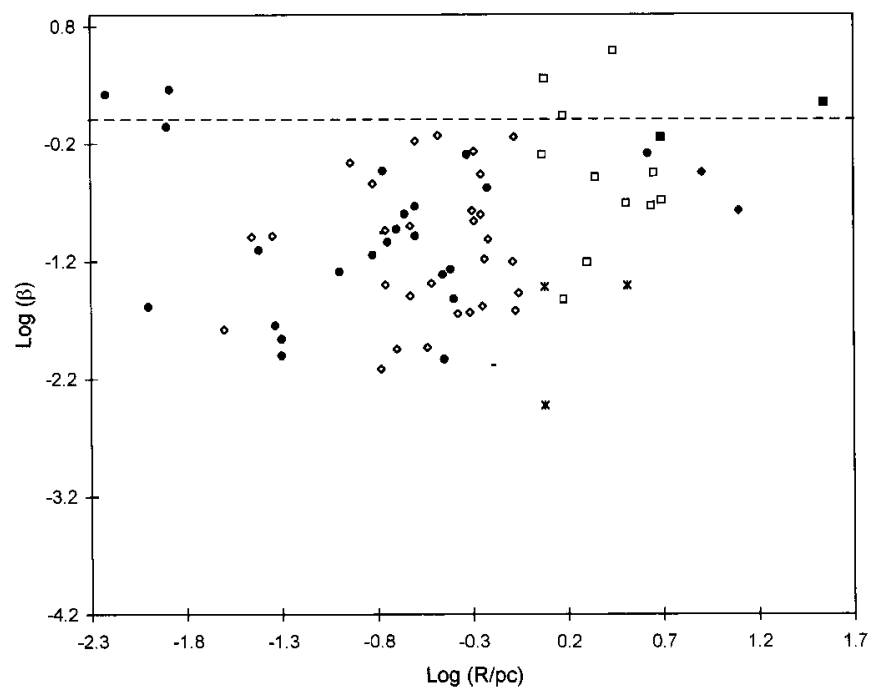

Fig. 8. Variation of the stability parameter $\beta(R)$ for isolated clouds and disks (see text for details). $\beta$ appears in most cases to be significantly less than unity, implying that turbulent and thermal virial expressions greatly exceed the contribution due to rotation

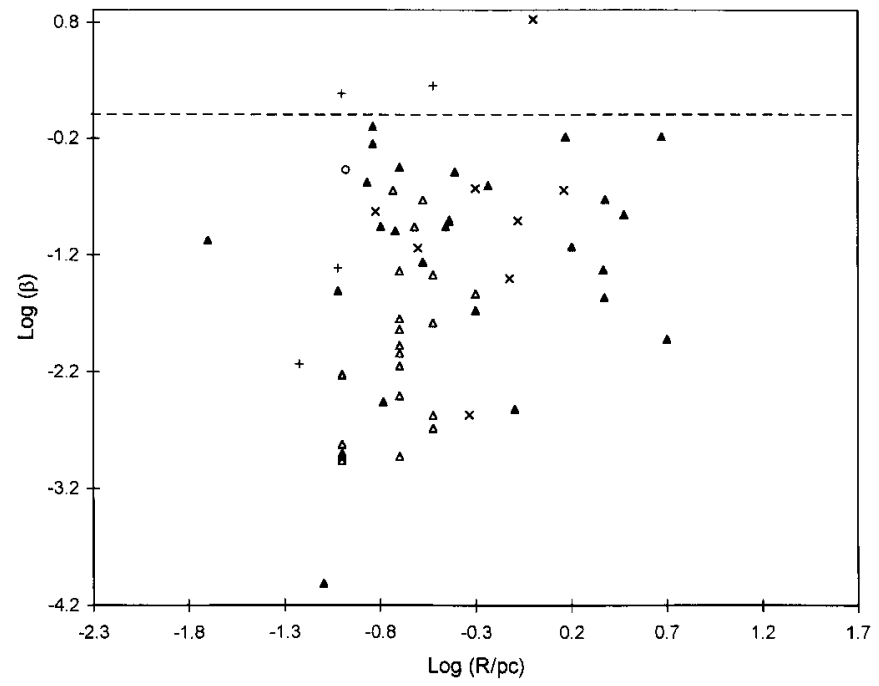

Fig. 9. Variation of the stability parameter $\beta(R)$ for clumps and condensations. Comparison with Fig. 8 suggests that rotation may be even less important for these sources than is the case for disks and larger cloud structures

where $\Delta V$ is the observed line FWHM. The influence of rotation and (primarily) turbulence are comparable when $\beta \cong 1$.

Values deriving from the present data are plotted in Figs. 8 and 9, where we have represented, separately, the distributions for clouds+disks and clumps.

It would appear, in both of these figures, that turbulence is by far the most critical factor in determining cloud stability; all but a handful of the results are appreciably less than unity (although the influence of turbulence appears rather stronger in the case of clumps).

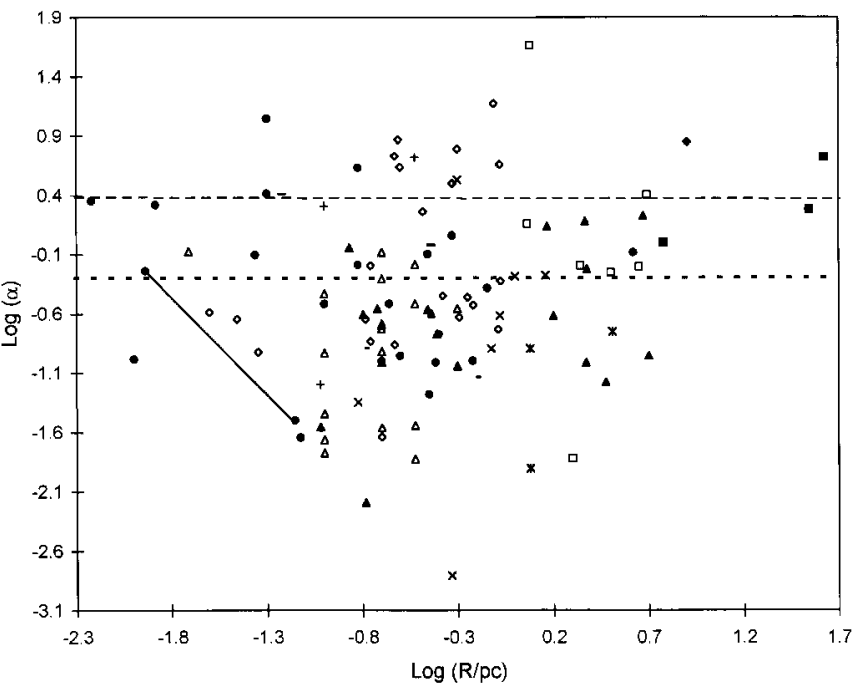

Fig. 10. Variation of the stability parameter $\alpha(R)$ for the complete data set (see text for details); where the lower dotted line corresponds to the parametric limit at which rotation becomes marginally significant. The upper dashed line represents the limiting value of $\alpha$ for highly flattened rotating disks

Certain caveats should be expressed at this point, however. The first is that in measuring projected velocity gradients $\mathrm{d} v / \mathrm{d} r$ we are in fact determining a parameter $\Omega \sin (i) \leq \Omega$, where $i$ is the inclination of vector $\Omega$ to the line of sight. Values of $\beta$ will therefore tend to be depressed by a factor $\sin ^{2}(i)$. A second qualification concerns the values of line width $\Delta V$ used to determine combined thermal and turbulent support. We shall discuss this in rather more detail later this section; suffice to say that observed line widths $\Delta V$ may also lead to an overestimate of kinematic broadening.

Taken as a whole, therefore, it would appear that such an analysis is likely to provide a somewhat pessimistic impression of the importance of cloud rotation for overall cloud stability.

A differing measure of the influence of cloud rotation upon cloud stability is provided through the parameter

$\alpha=22.0\left[\frac{\Omega}{10^{-4} \mathrm{~S}}\right]^{2}\left[\frac{R}{\mathrm{pc}}\right]^{3}\left[\frac{M}{M_{\odot}}\right]$

which represents a ratio between rotational and gravitational virial terms. Where $\alpha$ is less than 0.5 , then the influence of rotation may be regarded as small (e.g. McKee et al. 1993).

The distribution of values $\alpha$ for the present sample of nebulae is indicated in Fig. 10. We have also indicated (by a dashed line) an upper limit $\alpha=3 \pi / 4$ relevant for highly flattened, rotationally stabilised disks. On the other hand, if one represents filamentary clouds in terms of a cylinder of diameter $d$, length $L$, and density $\rho$, then the gravitational acceleration at either end of the filament would be $\pi G \rho d$. Rotational stability at the limits of the filament would then require values $\alpha=L / d=\Gamma$. It would appear, 
therefore, that $\alpha$ may approach values $>4$, based on the observed aspect ratios of filamentary clouds in the present study.

The results in Fig. 10 can be very broadly separated into three radial ranges. For sources having $R>2 \mathrm{pc}$, or $R<0.06 \mathrm{pc}$ (primarily disks), it appears that rotation is frequently important in maintaining overall stability. For intermediate radii, on the other hand, significantly fewer sources (approximately 1/3) appear to be influenced by rotation; although since $\alpha$ depends upon cloud inclination through $\sin ^{2}(i)$, it follows that the actual proportion of rotationally stabilised clouds may be significantly greater.

This data may also be sliced in a slightly different way. Thus, and taken as a whole, the individual clouds and disks appear to be strongly influenced by rotation in $\sim 50 \%$ of cases $(\sim 64 \%$ where clouds alone are considered), whilst the clumps possess $\alpha>0.5$ in only $\sim 31 \%$ of cases. As was found for $\beta$, therefore, it appears that clumps and condensations are less susceptible to the influence of rotation than is the case for other subgroups in this study.

In summary, it would appear that $\beta$ and $\alpha$ give somewhat differing impressions concerning the importance of cloud rotation. This, in part, may arise from the differing natures of the parameters we are evaluating, although $\beta$ also appears to be prone to systematic errors, and is likely (on average) to be appreciably undervalued.

Given that $\beta \propto \Delta V^{-2} \propto M_{\text {vir }}^{-1}$, we can further investigate the reasons for the depressed values of $\beta$ through an analysis of virial mass $M_{\mathrm{vir}}$. Thus, for instance, Fig. 11 illustrates the variation of virial mass (evaluated using linewidths quoted in Table 1) against corresponding column density mass estimates derived from the literature. It seems clear, from this, that there is a systematic disparity between the two comparative mass estimates, in the sense that values $M_{\text {vir }}$ are larger by a factor $\sim 4.8$; similar trends have also been noted by Zimmerman \& Ungerechts (1990), Keto \& Myers (1986), Stutzki \& Gusten (1990), Zhou et al. (1994) and Magnani et al. (1985) for other cloud samples. Various physical explanations might be sought for this difference, including pressure stabilisation by the ambient medium; the possibility that certain of these features are transient; or finally, that apparent line widths are somewhat broadened by line saturation - a problem which is by no means completely eliminated through our use of more optically thin transitions.

Whatever the reasons, it seems clear that observed line widths are rather larger than would be consistent with virial equilibrium, and this leads to a reduction in $\beta$ by $\sim 0.7$ dex. The parameter $\alpha$ would, under these circumstances, represent a rather more credible indicator of cloud stability.

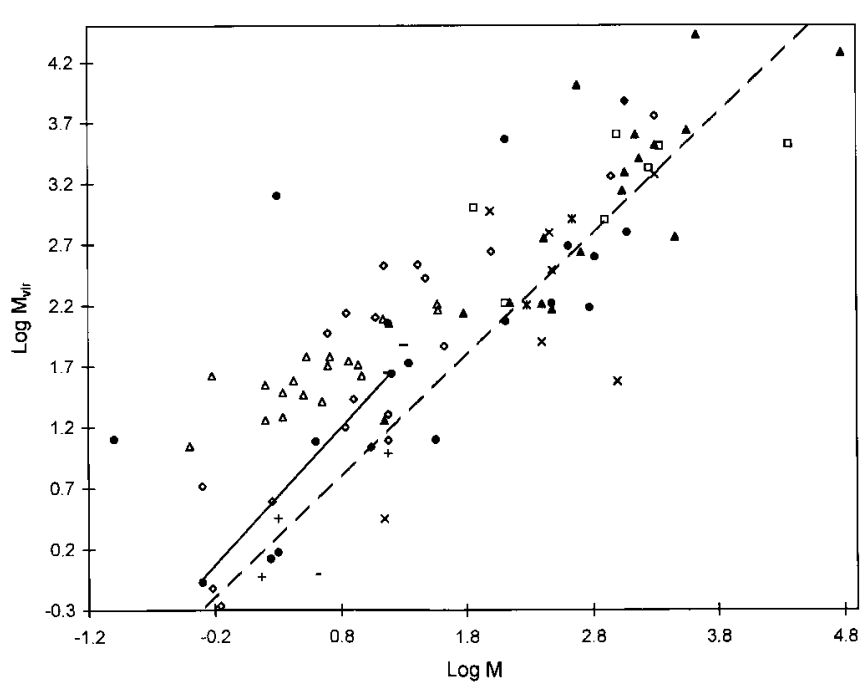

Fig. 11. Comparison of virial masses $M_{\text {vir }}$ deduced using line widths from Table 1 with cloud masses $M$ derived from molecular column densities. The diagonal dashed line represents the relationship which would be anticipated were clouds stable, line widths unaffected by saturation, and turbulent and thermal velocities constituted the dominant virial components
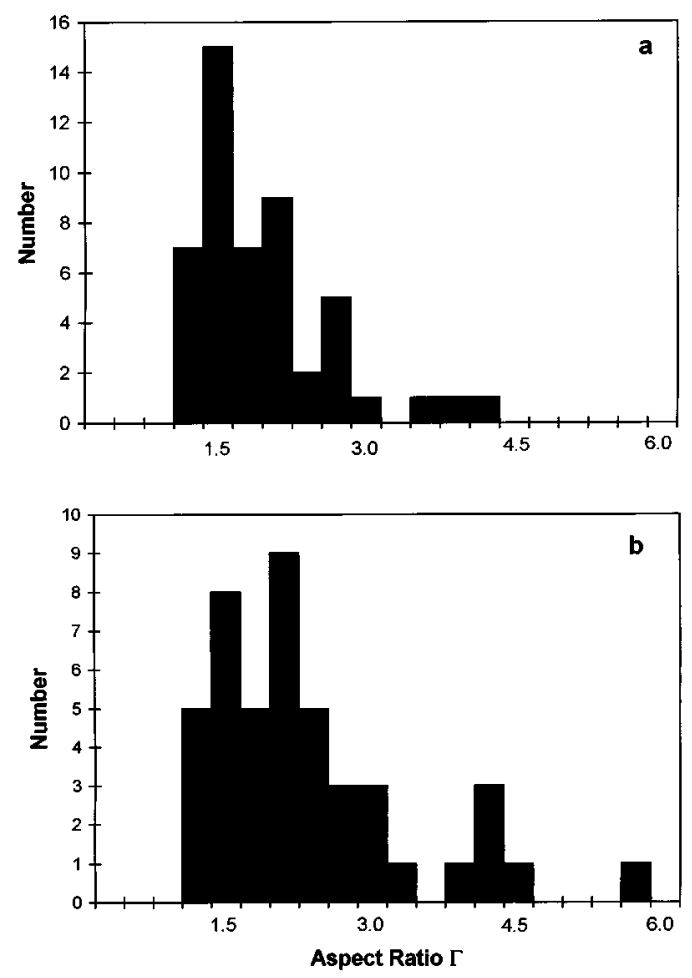

Fig. 12. Distribution of cloud aspect ratios $\Gamma$ for the stability parameters $\alpha \leq 0.3 \mathbf{a})$ and $\alpha>0.3 \mathbf{b}$ ). Note the broader distribution in $\Gamma$ for larger values of $\alpha$ 

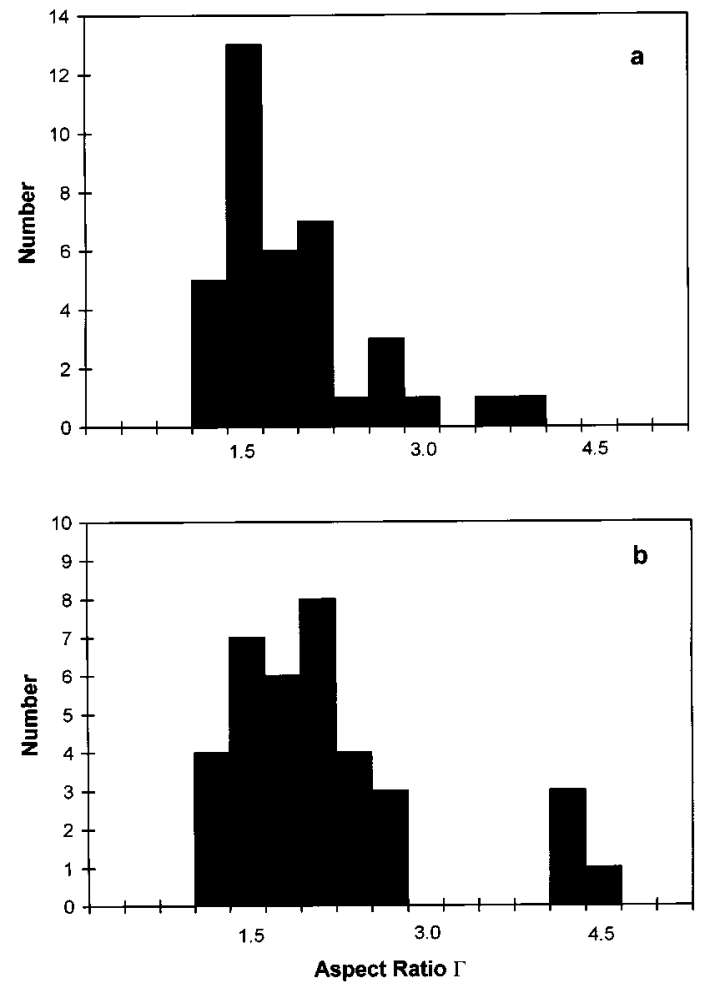

Fig. 13. Distribution of cloud aspect ratios $\Gamma$ for the stability parameters $\beta \leq 0.1$ a) and $\beta>0.1 \mathbf{b}$ ). Note the broader distribution in $\Gamma$ for larger values of $\beta$

\subsection{Cloud aspect ratios}

An interesting and related question is that of how strongly rotation affects the apparent aspect ratios of molecular clouds. Put at its most basic, one might anticipate that rapidly rotating structures would show typically higher aspect ratios than slowly rotating clouds. Thus, Miyama et al. (1984) find that aspect ratios for isothermal rotating disks are of order

$\Gamma=k\left[\frac{G M}{(J / M) c}\right]^{2}$

providing rotational forces at the cloud boundary exceed thermal support; where $c$ is the velocity of sound, and $k$ is a constant of order unity. This expression can also be generalised to include the combined turbulent and thermal pressure terms, whence for observed FWHM linewidths $\Delta V$ we determine

$$
\begin{aligned}
& \Gamma=1.0310^{-4}\left[\frac{M}{M_{\odot}}\right]^{2}\left[\frac{J / M}{\mathrm{~km} \mathrm{~s}^{-1} \mathrm{pc}}\right]^{-2}\left[\frac{\Delta V}{\mathrm{~km} \mathrm{~s}^{-1}}\right]^{-2} \\
& \approx 4.31\left[\frac{M}{M_{\odot}}\right]^{0.6}\left[\frac{\Delta V}{\mathrm{~km} \mathrm{~s}^{-1}}\right]^{-2}
\end{aligned}
$$

providing the stability parameter $\beta>1$, where we have substituted the trend for $J / M(M)$ from Table 2 . For reasonable mean line widths $\Delta V \sim 2 \mathrm{~km} \mathrm{~s}^{-1}$ it is therefore apparent that disks, and indeed clouds taken generally may show appreciable departures from sphericity. On the other hand, numerical simulations of comparable isothermal structures indicate that whilst $\Gamma$ is likely to increase with increasing $J / M$, the change overall may be comparatively modest (Boss 1987). Similarly, Myers et al. (1991) have noted that observed aspect ratios in dark clouds imply levels of rotational gradient which are not observed.

Do the present results offer any help in resolving this question? The answer, on the whole, is unclear. Thus, we note that the present sample of clouds appear to have values $\Omega$ oriented close to the minor axis in $\sim 75 \%$ of cases (Table 1, Col. 13); a result which is certainly consistent with rotational deformation.

Similarly, if we take separately the sources having $\alpha \leq 0.3$ and $\alpha>0.3$, and plot the corresponding distributions of aspect ratio $\Gamma$ (Fig. 12), then it seems that $\Gamma$ is larger for high values of $\alpha$ than is the case for clouds having lower values of $\alpha$. In particular, Gaussian fits to these distributions would suggest a width for the $\alpha>0.3$ values some $53 \%$ greater than for sources having lower values of $\alpha$, whilst very similar results are found for sources having $\beta \geq 0.1$ and $\beta<0.1$ (Fig. 13). On the other hand, the sample numbers are somewhat restricted, and it is not possible to claim a very high level of significance for these trends.

It would therefore seem that the present analysis offers some possible hint of rotational deformation, although further analyses (using larger samples) are necessary to establish any clear relationship between $\Omega$ and $\Gamma$.

\section{Discussion}

Several important trends have been identified as a result of the analysis of cloud rotational data in Sect. 3. It seems clear, in the first place, that angular velocity vectors in isolated clouds and complexes show a preferential orientation with respect to the galactic plane; a feature which would support an origin for cloud angular momenta in galactic rotation. This trend is not however shared by clumps and condensations within the clouds. Secondly, it appears that cloud rotation may be important for the stability of a surprisingly large number of these clouds, and influence observed aspect ratios. Thirdly, it appears that the trends for angular velocity and specific angular momentum are inconsistent with angular momentum conservation, turbulent vorticity, and models of axisymmetric, isothermal, non-magnetic clouds. Finally, it appears that angular momentum vectors for rings and (principally) disks possess a tendency towards non-random orientation, whilst rotational stabilisation is likely also to be important; characteristics which are to be found in neither clumps nor condensations. It is also notable that trends in $J / M, J$ and $\Omega$ with cloud radius $R$ differ from those of other sub-groups in this study.

It would appear, in brief, that disks are generically different from most other clouds investigated here; a 
feature which may argue for a physically distinct evolutionary sequence and, in all probability, reflects their differing spatio-kinematic structures. It is apparent, for instance, that these sources not only display typically large aspect ratios $\Gamma$ (although intrinsic values of this parameter are likely to be even greater), but also that angular velocities are among the most extreme, and the proportionate mass of embedded stars $M_{\text {ast }} / M$ is substantial (viz. Table 1). As a result, and more than for any other cloud subgroup, disk momentum depends upon the proportion of angular momentum carried by the stellar mass fraction, and the degree of angular momentum transfer that has occurred during protostellar contraction and subsequent evolution.

Although we have previously noted that turbulent vorticity gradients appear too large to explain observed overall trends in $\Omega(R)$, this is less the case for certain cloud subgroups. In particular, the variation in $\operatorname{dln} \Omega / \operatorname{d} \ln R=$ -0.67 predicted by Fleck \& Clark (1981) is not too different from that estimated for clouds $(\cong-0.53)$ and condensations $(\cong-0.60)$ taken separately.

The theory of Fleck and Clark requires however to be somewhat revised. In particular, these authors assume strict energy cascade from large to small turbulent eddy scales, whence turbulent velocity $V_{\text {turb }} \propto R^{0.33}$. In reality, however, it seems that most of the clouds in this study are likely to be characterised by supersonic turbulence (e.g. Myers 1983), and compressible fluid interactions, whence $V_{\text {turb }}(R)$ may depart considerably from the trend predicted by Kolmogoroff theory (Fleck 1983).

If, in place of this, one therefore employs the observed relation $V_{\text {turb }} \propto R^{0.5}$ (see for instance Myers (1983), and also the recent commentary by Phillips 1998), it is then possible to evaluate a revised vorticity gradient $\mathrm{d} \ln \Omega / \mathrm{d} \ln R=-0.5$.

Although this latter parameter is somewhat greater than supposed before, it would still (within uncertainties) be consistent with the relations noted for clouds and condensations. In the case of condensations alone, therefore, it may be possible to comprehend observed variations in rotational parameters in terms of turbulent interactions. Such an explanation is likely to be less relevant in the case of isolated clouds and complexes, however, given the orientational disposition of their vectors $\Omega$.

It may also, on the other hand, be possible to explain certain of the functional trends in rotation in terms of magnetic braking. Thus, where cloud fields are appreciably greater than in the intercloud medium, and the density contrast between cloud and exterior medium is given by $\rho_{\mathrm{c}} / \rho_{\mathrm{m}}$, angular momentum would be transferred outwards over a time-scale (Gillis et al. 1974, 1979; Mestel \& Paris 1979, 1984)

$\tau_{\omega} \cong 0.27\left[\frac{M^{2} G a}{B^{2} R^{4} b}\right]^{0.5}\left[\frac{\rho_{\mathrm{c}}}{\rho_{\mathrm{m}}}\right]^{0.1} \tau_{\mathrm{ff}}$ where the free-fall timescale

$\tau_{\mathrm{ff}}=\left[\frac{3 \pi}{32 G \rho_{\mathrm{c}}}\right]^{0.5}$

and parameter $b=0.75$ for a sphere and $\pi^{-1}$ for a disk. The value of a is given by $(1-\gamma / 3) /(1-2 \gamma / 5)$ for a radial cloud density variation $\rho(r) \propto r^{-\gamma}$; we shall simply assume here a value $a=1$, appropriate for spheres with uniform density. Substituting $\rho_{\mathrm{c}} / \rho_{\mathrm{m}} \sim 10$, and $B \cong 12 \mu \mathrm{G}$ (Phillips 1998) then yields $\tau_{\omega} \cong 2.4510^{4}\left(M / M_{\odot}\right)^{0.5}$ $(R / \mathrm{pc})^{-0.5} \mathrm{yrs} \cong 3.810^{5}(R / \mathrm{pc})^{0.23} \mathrm{yrs}$.

How does this compare with observations? The first characteristic to note is that the gradient $\mathrm{d} \ln (J / M) / \mathrm{d} M \cong 0.7$ is actually less than would be predicted for isothermal models of non-magnetic rotating clouds. If one assumes, on the other hand, that the logarithmic decrement in $J / M$ arises primarily from magnetic braking, and that there is an evolutionary trend from large to small cloud structures, then a fractional reduction in specific angular momentum $1-f=\delta(J / M) /(J / M)=0.7$ would imply comparatively large radial changes $\Delta R / R=$ $\left(1-f^{0.79}\right) \cong 0.6$. It is apparent, in brief, that the decrement in $J / M$ is not only uniform and continuous in $R$ and $M$, but appears also to be comparatively shallow; we perceive no evidence for the strong magnetic braking which has been presumed heretofore.

Several routes might be suggested whereby this problem could be side-stepped. Thus, it is possible to assume that values $B$ have been greatly overestimated; a presumption which seems unlikely given the observations of this parameter through Zeeman splitting (cf. Myers \& Goodman 1988). An alternative possibility is that a large fraction of clouds are magnetically supercritical, and possess density contrasts $\rho_{\mathrm{c}} / \rho_{\mathrm{m}}>10$, leading to reduced values of $\tau_{\omega}$ (McKee et al. 1993); a situation which may be particularly applicable to GMC's (McKee 1989).

Finally, we note that cloud contraction velocities $V_{\mathrm{c}} \geq$ $V_{\nu}$ (where $V_{\nu}$ is the Alfven velocity) would lead to the trapping of Alfven waves (Mouschovias 1989), and a considerable reduction in the rate of angular momentum transfer. In particular, given that

$\frac{V_{\nu}}{\mathrm{km} \mathrm{s}^{-1}}=\frac{B}{\left(4 \pi \rho_{\mathrm{c}}\right)^{0.5}} \cong 7.0210^{5} \frac{B(R / \mathrm{pc})^{1.5}}{\left(M / M_{\odot}\right)^{0.5}}$

then for $B \sim 12 \mu \mathrm{G}$ we obtain $V_{\nu}=0.55$ $(R / \mathrm{pc})^{0.78} \mathrm{~km} \mathrm{~s}^{-1}$ (where we have also substituted for $M(R)$ from Table 2$)$. Given also that free-fall collapse velocities $V_{\mathrm{ff}}=(2 G M / R)^{0.5} \cong 1.42(R / \mathrm{pc})^{0.23} \mathrm{~km} \mathrm{~s}^{-1}$, then $V_{\mathrm{ff}} \geq V_{\nu}$ for $R \leq 1.7 \mathrm{pc}$. Trapping may therefore be possible over comparatively short periods, where contraction occurs at velocities comparable or greater than the free fall velocity $V_{\mathrm{ff}}$ - a situation which may, in turn, arise through external cloud compression by winds and expanding shells (e.g. Elmegreen \& Lada 1977; although note that radial decrements in $V_{\nu}$ are likely to be less steep given a dependency $B \propto \rho_{\alpha}, 1 / 3 \leq \alpha \leq 1 / 2$ (Gordon 1988)). The mean 
velocity of contraction for clouds taken as a whole, on the other hand, is on the order of $\left\langle V_{\mathrm{c}}\right\rangle=R_{0} m_{\mathrm{ast}} / \varepsilon M_{\mathrm{cx}} \cong$ $0.34 \mathrm{~km} \mathrm{~s}^{-1}$, where $m_{\text {ast }} \cong 3 M_{\odot} \mathrm{yr}^{-1}$ is the mean galactic rate of star formation, $\varepsilon \cong 0.1$ is the efficiency of star formation (Vrba 1977), and $M_{\mathrm{cx}} \cong 410^{9} M_{\odot}$ is the mass of gas in the form of molecular complexes (Solomon \& Sanders 1980). The parameter $\left\langle V_{\mathrm{c}}\right\rangle$ (which may be regarded as lower limit velocity for quiescent and continuous contraction) is therefore only a little less than $V_{\nu}$ for moderately sized clouds.

It is therefore conceivable that a trapping mechanism of kind noted above may indeed operate to reduce rates of angular momentum transfer; although the effectiveness of this process is undoubtedly enhanced where contraction proceeds in fits and starts, rather than through a uniform global decrease in radius.

Finally, we have failed in the above discussion to address several outstanding questions, including the unusually close correlation between angular momentum $J$ and non-virial cloud mass $M$ (Fig. 6), and the strange disparity in rotational properties between disks, and both clumps/condensations and larger cloud structures. In this latter respect, we note that one conceivable solution to the (possible) orientational disparity between clumps/condensations and disks may be that clumps, after all, are not rotating; that despite the attribution of rotation to these features in the literature, we are in fact (say) witnessing the superposition of differing, unrelated features. A problem with this supposition, however, is that the broad trends in $\Omega, J$, and $J / M$ noted for clumps and condensations are also closely similar to those determined for clouds - and not too far removed from those for disks as well (although to be sure, disks are in this respect somewhat different from all other subgroups). Such a correspondence would not be anticipated were gradients to arise from random clump superposition.

This can be expressed in a slightly more precise way. Thus, if we assume that projected clump radii $\Delta R$ are, in the mean, invariant with cloud size, and that the mean separation between unresolved clump-pairs is also constant (consistent with the approximate invariance in cloud column densities), then the typical angular velocity attributed to such superpositions would be $\Omega \propto \sigma_{\text {turb }} / \Delta R \propto$ $\sigma_{\text {turb }}$ (where $\sigma_{\text {turb }}$ is the line of sight velocity dispersion due to turbulence). If we assume virial stability, and employ a relation $\operatorname{dnn} M_{\mathrm{vir}} / \mathrm{d} \ln M=0.73$ based on a least squares analysis of the present results (thermal velocity contributions to line width can normally be regarded as small, and have been ignored (viz. Myers 1983)), then cloud mass $M \propto \sigma_{\text {turb }}^{2.56}$. As a consequence, one would then anticipate $\Omega \propto M^{0.37}$, and (given $M \propto R^{0.78}$ from Table 2) $J \propto M^{3.9}$. These relations are not consistent with the trends noted in Sect. 3.

On the other hand, it is by no means unreasonable that clump orientations should be so disordered; supersonic and magnetic interactions are sufficient to ensure appreciable orientational randomisation of the angular velocity vectors $\Omega$. The real mystery, in all probability, is why fossil echoes of prior orientations should be retained by disk-like structures alone.

\section{Conclusions}

We have compiled a large data base of rotational measures for a broad range of molecular cloud types, derived from observations of velocity gradients taken primarily at millimetric wavelengths. As a consequence, it has proved possible to undertake a detailed investigation of cloud rotational parameters, and to evaluate the importance of angular momentum in maintaining cloud stability.

We have demonstrated that two measures of stability based on the ratio between angular momentum and turbulent/thermal virial terms (parameter $\beta$ ), and between rotation and gravity $(\alpha)$ lead to somewhat differing conclusions. Given the potentially large systematic errors to which $\beta$ is prone, however, it seems likely that a substantial fraction of clouds may be appreciably stabilised by rotation. Employing only parameter $\alpha$, for instance, we find that $\sim 50 \%$ of clouds and disks are to some degree stabilised through rotation, although clumps and condensations appear more dependant upon turbulent support. High levels of angular momentum may also be responsible for departures in cloud sphericity, and we note that structures with large values of $\alpha$ and $\beta$ are characterised by typically larger aspect ratios $\Gamma$.

The orientation of $\Omega$ is found to vary markedly between various cloud groups, with clumps and condensations displaying a more-or-less random distribution with respect to the galactic plane. Isolated clouds (and perhaps disks), on the other hand, appear to favour orientations towards the north and south galactic poles.

These disparities presumably imply that rotation in larger cloud structures and disks derives from galactic shear, whilst vectors $\Omega$ for clumps and condensations have either been randomised through dynamical and/or magnetic interactions, or derive from turbulent vorticity.

A comparison between observed functional trends in $\Omega, J / M$, and $J$, and those predicted through models of cloud rotation has proven particularly interesting. Thus, it appears that derived trends in $J(M)$ are broadly similar to those predicted through simple clump merger models. On the other hand, the variation of $J / M$ with radius appears not to be consistent with models of isothermal rotating clouds, whilst $d \ln \Omega / d \ln R$ is inconsistent with either conservation of angular momentum or models of turbulent vorticity (although in this latter case, clumps and condensations follow predictive trends rather more closely than is the case for the sample as a whole). Whilst magnetic braking may account for at least some of the observed decrement in $J / M$, the present results imply gradients $\mathrm{d} \ln (J / M) / \mathrm{d} M$ which are less than would normally be expected through such a mechanism; a disparity which may 
arise through a variety of causes, including the trapping of Alfven waves during periods of rapid cloud contraction.

Whilst most of the functional trends for cloud rotation display moderately high levels of correlation, there appear also to be certain exceptions to this rule. Thus, the trend between $J$ and $M$ has a correlation coefficient $r=0.98$, whilst the coefficient for $\Omega(M)$ varies between 0.03 and 0.41 (depending upon cloud type). Whilst it seems likely that such differences must arise from the nature of angular momentum transfer from large to small scales, we have as yet little understanding of the root origins of these disparities.

We have, finally, noted that gradients $\mathrm{d} \ln \Omega / \mathrm{d} \ln R$, $\operatorname{dln}(J / M) / \operatorname{d} \ln R$, and $\operatorname{dn} M / \operatorname{d} \ln R$ for disks appear significantly different from those characterising most other cloud subgroups; a disparity which presumably derives from their markedly differing spatio-kinematic structures, and proportionately high fractional stellar mass contents.

\section{Appendix}

The parameters $J, \Omega, J / M, M$ and so forth depend to varying degrees upon distance $D$, such that (for instance) $\Omega \propto D^{-1}$, and $J / M \propto D$. It follows that errors in distance may give rise to correlations between rotational and physical parameters over and above those attributable to intrinsic cloud properties. How important are such effects for the sample investigated here?

To assess this, we have investigated a model containing $10^{3}$ clouds having intrinsic values $\log J, \log \Omega$ etc. distributed randomly within pre-set limits. These limits are in turn defined such that the final "observed" ranges in these parameters (including the effects of distance uncertainties) are comparable to those noted in Table 1, and Figs. 2-7. In particular, if the intrinsic logarithmic range in $J$ is given by $\Delta \log J_{\text {int }}$, and the range of values $J$ which would be determined through observation (and in the presence of distance errors) is $\Delta \log J_{\text {obs }}$, then $\Delta \log J_{\text {int }}$ is tailored to make $\Delta \log J_{\text {obs }} \sim 11$; comparable to the range noted in Figs. 6 and 7.

Errors in estimated distance are taken to be of a form:

$\log D_{\mathrm{obs}}=\log D_{\mathrm{int}}+\Sigma(R-0.5)$

where again $D_{\text {obs }}$ is the "observed" or estimated value of distance, $D_{\text {int }}$ is the intrinsic value of $D, \Sigma$ is a constant, and $R$ is a random number generator varying between 0 and 1 . The clouds, in brief, have estimated distances which vary randomly between limits $D_{\text {int }} 10^{ \pm 0.5 \Sigma}$ on either side of the actual distances. Similarly, the intrinsic range in cloud distances is restricted to $\Delta \log D_{\text {int }}=2-\Sigma$. Where $\Sigma=0$ then errors in estimated distance are negligible, and the range in $\log D_{\text {obs }}$ is comparable to the intrinsic range (i.e. $\Delta \log D_{\text {int }}=\Delta \log D_{\text {obs }}=2$ ). Where $\Sigma=2$ then the range in $D_{\text {obs }}$ is due almost entirely to errors.
Table 3. Correlation coefficients and gradients for simulated cloud trends

\begin{tabular}{lccccc}
\hline$F(y)$ & $F(x)$ & $b$ & \multicolumn{3}{c}{$r$} \\
\cline { 4 - 6 } & & & $\Sigma=0.5$ & $\Sigma=1.0$ & $\Sigma=1.5$ \\
\hline \hline $\log \Omega$ & $\log R$ & -0.33 & 0.03 & 0.14 & 0.33 \\
$\log \Omega$ & $\log M$ & -0.17 & 0.03 & 0.14 & 0.29 \\
$\log (J / M)$ & $\log M$ & 0.22 & 0.04 & 0.10 & 0.26 \\
$\log (J / M)$ & $\log R$ & 0.36 & 0.00 & 0.15 & 0.24 \\
$\log J$ & $\log M$ & 0.67 & 0.04 & 0.14 & 0.37 \\
$\log J$ & $\log R$ & 0.33 & 0.03 & 0.07 & 0.19 \\
\hline
\end{tabular}
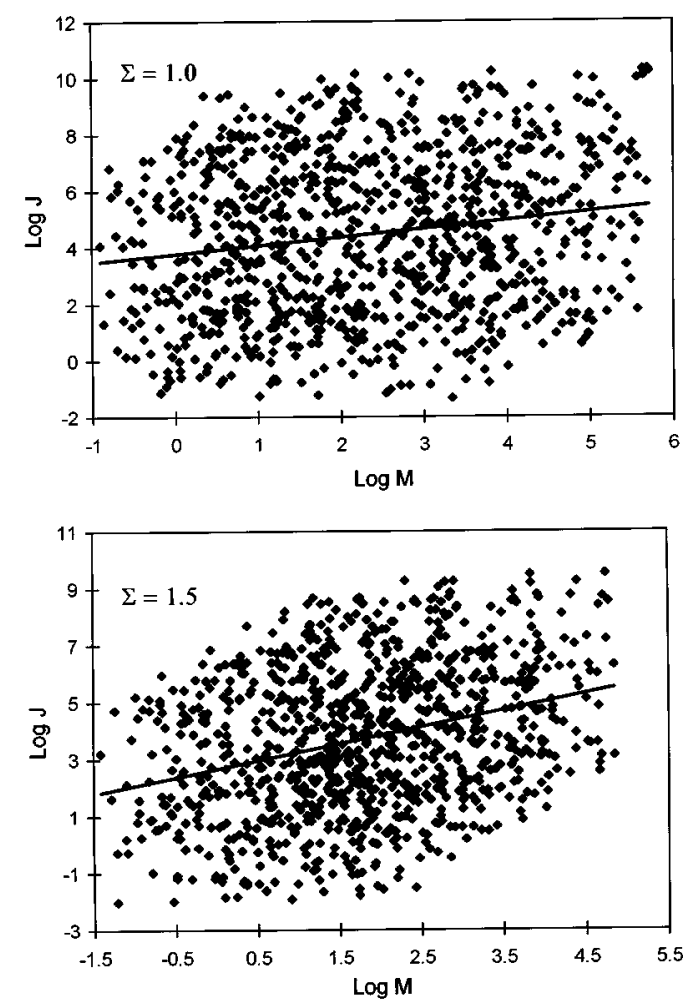

Fig. 14. Theoretical trends between $J$ and $M$ for a $10^{3}$ cloud sample, and two values of the distance error parameter $\Sigma$ (see appendix for details). The diagonal lines correspond to linear least-squares fits

Under these circumstances, it is possible to investigate how important such errors would be in creating spurious correlations, for a sample in which there is no initial correlation between the various cloud parameters (i.e. in which intrinsic values of $\Omega$, say, are uncorrelated with $M$, $J$ or any other parameter). A summary of the results is provided in Table 3, wherein we indicate correlation coefficients for various values of $\Sigma$, and relative parametric trends. The simulated variation between $J$ and $M$ is also indicated in Fig. 14 for two values of $\Sigma$.

It is clear, from these, that appreciable correlations may indeed result, but only where $\Sigma$ is large; and even for $\Sigma=1.5$, which would correspond to a factor 5.6 uncertainty in distance, it is apparent that values for $r$ are very much less than observed here (Table 2). Similarly, the gradients $b$ for these distributions depend upon the 
intrinsic ranges in the parameters, and error factor $\Sigma$. Even for the most extreme case investigated here (the gradients $b$ in Table 3 correspond to $\Sigma=1.5$ ) it is apparent that gradients are significantly different from what is observed (Table 2).

Given that errors in $D$ are almost certainly appreciably smaller, and $\Sigma$ is no more than $\sim 0.3 \Rightarrow 0.5$, we conclude that the influence of such uncertainties upon observed correlations is likely to be small.

\section{References}

Abraham P., Dobashi K., Mitzuno A., Fukui Y., 1995, A\&A 300,525

Arquilla R., Goldsmith P.F., 1985, ApJ 297, 436

Arquilla R., Goldsmith P.F., 1986, ApJ 303, 356

Bally J., 1982, ApJ 261, 558

Bally J., Castets A., Duvert G., 1994, ApJ 423, 310

Baudry A., Cernichero J., Pèrault M., Noë J. de la, Despois D., 1981, A\&A 104, 101

Beckwith S., Evans N.J., Becklin E.E., Neugebauer G., 1976, ApJ 208, 390

Benz W., 1984, A\&A 139, 378

Bieging J.H., 1984, ApJ 286, 591

Blitz L., 1993, in Protostars \& Planets III, Levy E.H. \& Lunine J.I. (eds.). Tucson: University of Arizona Press, p. 125

Blitz L., Thaddeus P., 1980, ApJ 241, 676

Bodenheimer P., Black D.C., 1987, in Protostars \& Planets, Gehrels T. (ed.). Tucson: University of Arizona Press, p. 288

Boss A.P., 1987, ApJ 316, 721

Brackmann E., Scoville N., 1980, ApJ 242, 112

Brooks J.W., Sinclair M.W., Manefield G.A., Goss W.M., 1976, MNRAS 177, 299

Casali M.M., 1986, MNRAS 223, 341

Casali M.M., Edgar, M.L., 1987, MNRAS 225, 481

Clark F.O., Johnson D.R., 1978, ApJ 220, 500

Clark F.O., Johnson D.R., 1981, ApJ 247, 104

Clemens D.P., Dickman R.L., Ciardi D.R., 1992, AJ 104, 2165

Crutcher R.M., 1973, Astrophys. Lett. 14, 147

Crutcher R.M., Hartkopf W.I., Giguere P.T., 1978, ApJ 226, 839

Davidson J.A., Jaffe D.T., 1984, 277, L13

Dickman R.L., Clemens D.P., 1983, ApJ 271, 143

Downes D., Genzel R., Becklin E.E., Wynn-Williams C.G., 1981, ApJ 244, 869

Duvert G., Cernichero J., Baudry A., 1986, A\&A 164, 349

Elmegreen D.M., Elmegreen B.G., 1979, AJ 84, 615

Elmegreen B.G., Lada C.J., 1977, ApJ 214, 725

Few R.W., Cohen R.J., 1983, MNRAS 203, 853

Field G.B., 1987, in Protostars \& Planets, Gehrels T. (ed.). Tucson: University of Arizona Press, p. 243

Fleck R.C., 1983, ApJ 272, L45

Fleck R.C., Clark F.O., 1981, ApJ 245, 898

Frerking M.A., Langer W.D., Wilson R.W., 1987, ApJ 313, 320 Gillis J., Mestel L., Paris R.B., 1974, Astrophys. Lett. 27, 167 Gillis J., Mestel L., Paris R.B., 1979, MNRAS 187, 311

Goldsmith P.F., Arquilla R., 1985, in Protostars and Planets II, de. Black D.C. and Matthews M.S. (eds.). Tucson: University of Arizona Press, p. 135
Gordon M.A., 1988, in Galactic and Extragalactic Radio Astronomy, Verschuur G.L. \& Kellermann K.I. (eds.). Berlin: Springer-Verlag, p. 37

Guilloteau S., Forveille T., Baudry A., Despois D., Goss W.M., 1988, A\&A 202, 189

Goldsmith P.F., Sernyak M.J., 1984, ApJ 283, 140

Gonzlez-Alfonso E., Cernicharo J., Radford S.J.E., 1995, A\&A 293, 493

Gusten R., Chini R., Neckel T., 1984, A\&A 138, 205

Gusten R., Marcaide J.M., 1986, A\&A 164, 342

Hanawa T., Nakamura F., Matsumoto T., et al., 1993, ApJ 404, L83

Harjunpaä P., Liljeström T., Mattila K., 1991, A\&A 249, 493

Harris A., Townes C.H., Matsakis D.N., Palmer P., 1983, ApJ 265, L63

Harvey P.M., Campbell M.F., Hoffmann W.F., Thronson H.A., Gatley I., 1979, ApJ 229, 990

Harvey P.M., Gatley I., Werner M.W., et al., 1974, ApJ 189, L87

Hasegawa T., Kaifu N., Inatani J., et al., 1984, ApJ 283, 117

Heiles C., Katz G., 1976, AJ 81, 37

Hayashi M., Kobayashi H., Hasegawa T., 1989, ApJ 340, 298

Heyer M.H., 1988, ApJ 324, 311

Heyer M.H., Snell R.L., Goldsmith P.F., Strom S.E., Strom K.M., 1986, ApJ 308, 134

Heyer M.H., Vrba F.J., Snell R.L., et al., 1987, ApJ 321, 855

Ho P.T.P., Haschick A.D., 1986, ApJ 304, 501

Ho P.T.P., Tereby S., Turner J.L., 1994, ApJ 423, 320

Iwata T., Fukui Y., Ogawa H., 1987, ApJ 325, 372

Jackson J.M., Ho P.T.P., Haschick A.D., 1988, ApJ 333, L73

Kaifu N., Suzuki S., Hasegawa T., et al., 1984, A\&A 134, 7

Mouschovias T., 1989, in Physical Processes in Interstellar Clouds, Winnewisser G. \& Armstrong J.T. (eds.). Berlin: Springer-Verlag, p. 297

Kastner J.H., Weintraub D.A., Snell R.L., et al., 1994, ApJ 425,695

Kazuhito D., Yonekura Y., Mizuno A., Fukui Y., 1992, AJ 104, 1525

Keto E.R., Ho P.T.P., Haschick A.D., 1987, ApJ 318, 712

Keto E.R., Ho P.T.P., Haschick A.D., 1988, ApJ 324, 920

Keto E.R., Ho P.T.P., Reid M.J., 1987, ApJ 323, L117

Keto E.R., Myers P.C., 1986, ApJ 304, 466

Kawabe R., Ogawa H., Fukui Y., et al., 1984, ApJ 282, L69

Kislyakov A.G., Turner B.E., 1976, AJ 81, 302

Kleiner S.C., Dickman R.L., 1982, ApJ 295, 466

Kutner M.L., Evans N.J., Tucker K.D., 1976, ApJ 209, 452

Kutner M.L., Tucker K.D., 1975, ApJ 199, 79

Kutner M.L., Tucker K.D., Chin G., Thaddeus P., 1977, ApJ 215,521

Lada C.J., Gull T.R., Gottlieb C.A., Gottlieb E.W., 1976, ApJ 203, 159

Lada C.J., Thronson H.A., Smith H.A., Schwartz P.R., Glaccum W., 1984, ApJ 286, 302

Lang K.R., Willson R.F., 1980, ApJ 238, 867

Lehtinen K., Mattila K., Schnur G.F.O., Prusti T., 1995, A\&A 295,487

Leung C.M., Kutner M.L., Mead K.N., 1982, ApJ 262, 583

Lichten S.M., 1982, ApJ 253, 593

Liljeström T., 1989, A\&A 219, L19

Linke R.A., Wannier P.G., 1974, ApJ 193, L41

Lis D.C., Goldsmith P.F., Carlstrom J.E., Scoville N.Z., 1993, ApJ 402, 238 
Little L.T., Brown A.T., Macdonald G.H., Riley P.W., Matheson D.N., 1980, MNRAS 193, 115

Little L.T., Dent W.R.F., Heaton B., Davies S.R., White G.J., 1985, MNRAS 217, 227

Loren R.B., 1977a, ApJ 215, 129

Loren R.B., 1977b, ApJ 218, 716

Loren R.B., Sandqvist A.A., Wootten A., 1983, ApJ 270, 620

Machnick D.E., Hettrick M.C., Kutner M.L., Dickman R.L., Tucker K.D., 1980, ApJ 242, 121

Magnani L,. Blitz L., Mundy L., 1985, ApJ 295, 402

Martin R.N., Barrett A.H., 1978, ApJS 36, 1

Mathieu R.D., Benson P.J., Fuller G.A., Myers P.C., Schild R.E., 1988, ApJ 330, 385

Matsakis D.N., Bologna J.M., Schwartz P.R., Cheung A.C., Townes C.H., 1980, ApJ 241, 655

McCutcheon W.H., Roger R.S., Dickman R.L., 1982, ApJ 256, 139

McCutcheon W.H., Vrba F.J., Dickman R.L., Clemens D.P., 1986, ApJ 309, 619

McKee C.F., 1989, ApJ 345, 782

McKee C.F., Zweibel E.G., Goodman A.A., Heiles C., 1993, in Protostars \& Planets III, Levy E.H. \& Lunine J.I. (eds.). Tucson: University of Arizona Press, p. 327

Menten K.M., Harju J., Olano C.A., Walmsley C.M., 1989, A\&A 223, 258

Menten K.M., Serabyn E., Güsten R., Wilson T.L., 1987, A\&A 177, L57

Menten K.M., Walmsley C.M., Krügel E., Ungerechts H., 1984, A\&A 137, 108

Mestel L., Paris R.B., 1979, MNRAS 187, 337

Mestel L., Paris R.B., 1984, A\&A 136, 98

Milman A.S., 1977, ApJ 211, 128

Miyama S.M., Hayashi C., Narita S., 1984, ApJ 279, 621

Mundy L.G., Cornwell T.J., Masson C.R., et al., 1988, ApJ 325,382

Myers P.C., 1983, ApJ 270, 105

Myers P.C., Benson P.J., 1983, ApJ 266, 309

Myers P.C., Fuller G.A., Goodman A.A., Benson P.J., 1991, ApJ 376, 561

Myers P.C., Goodman A.A., 1988, ApJ 326, L27

Myers P.C., Ho P.T.P., 1975, ApJ 202, L25

Nachman P., 1979, ApJS 39, 103

Nakamura A., Kawabe R., Kitamura Y., et al., 1991, ApJ 383, L81

Nercessian E., Castets A., Cernichero J., Benayoun J.J., 1988, A\&A 189, 207

Nozawa S., Mizuno A., Teshima Y., Ogawa H., Fukui Y., 1991, ApJS, 77, 647

Patel N.A., Xie T., Goldsmith P.E., 1993, ApJ 413, 593

Phillips J.P., 1998 (submitted for publication)

Phillips J.P., Mampaso A., 1991, A\&AS, 88, 189

Phillips J.P., Mampaso A., 1996, A\&A 316, 182

Phillips J.P., White G.J., 1983, MNRAS 202, 1093

Phillips J.P., White G.J., Rainey R., et al., 1988, A\&A 190, 237

Rickard L.J., Palmer P., Buhl D., Zuckerman B., 1977, ApJ 213,654

Schloerb P.F., Snell R.L., 1984, ApJ 283, 129

Scoville N.Z., Sargent A.I., Sanders D.B., et al., 1986, ApJ 303,
416

Snell R.L., 1981, ApJS 45, 121

Snell R.L., Edwards S., 1982, ApJ 259, 668

Solomon P.M., Sanders D.B., 1980, in Giant Molecular Clouds in the Galaxy, Solomon P.M. \& Edmunds M.G. (eds.). New York: Pergamon Press

Stutzki J., Gusten R., 1990, ApJ 356, 513

Sume A., Downes D., Wilson T.L., 1975, A\&A 39, 435

Sutton E.C., Danchi W.C., Jaminet P.A., Masson C.R., 1990, ApJ 348, 503

Tafalla M., Bachiller R., Martin-Pintado J., 1993, ApJ 403, 175

Takano T., Fukui Y., Ogawa H., et al., 1984, ApJ 282, L69

Takano T., Stutzki J., Fukui Y., Winnewisser,G., 1986a, A\&A 158,14

Takano T., Stutzki J., Fukui Y., Winnewisser G., 1986b, A\&A 167,333

Thronson H.A., Lada C., Hewagama T., 1985, ApJ 297, 662

Tieftrunk A.R., Wilson T.L., Steppe H., et al., 1995, A\&A 303, 901

Tölle F., Ungerechts H., Walmsley C.M., Winnewisser G., Churchwell E., 1981, A\&A 95, 143

Torrelles J.M., Ho P.T.P., Moran J.M., Rodriguez L.F., Canto J., 1986, ApJ 307, 787

Torrelles J., Ho P.T.P., Rodriguez L.F., Canto J., 1986, ApJ 305,721

Torrelles J.M., Rodriguez L.F., Canto J., Ho P.T.P., 1993, ApJ 404, L75

Torrelles J.M., Rodriguez L.F., Canto J., et al., 1983, ApJ 274, 214

Ungerechts H., Walmsley C.M., Winnewisser G., 1982, A\&A 111,339

Vallee J.P., Avery L.W., 1990, A\&A 233, 553

Vogel S.N., Bieging J.H., Plambeck R.L., Welch W.J., Wright M.C.H., 1985, ApJ 296, 600

Vogel S.N., Welch W.J., 1983, ApJ 269, 568

Vrba F.J., 1977, AJ 82, 198

Wang T., Wouterloot J.G.A., Wilson T.L., 1993, A\&A 277, 205

White G.J., Phillips J.P., 1981, MNRAS 194, 947

Willson R.F., Folch-Pi F.J., 1981, AJ 86, 1084

Wilson T.L., Serabyn E., Henkel C., Walmsley C.M., 1986, A\&A 158, L1

Wootten A., Loren R.B., Sandqvist A., Friberg P., Hjalmarson A., 1984, ApJ 279, 633

Wouterloot J.G.A., 1984, A\&A 134, 244

Yang J., Fukui Y., Umemoto T., Ogawa H., 1990, ApJ 362, 538

Young J.S., Goldsmith P.F., Langer W.D., Wilson R.W., Carlson E.K., 1982, ApJ 261, 513

Young J.S., Langer W.D., Goldsmith P.F., Wilson R.W., 1981, ApJ 251, L81

Zheng X.W., Ho P.T.P., Reid M.J., Schneps M.H., 1985, ApJ 293, 522

Zhou S., Evans N.J., Wang Y., Peng R., Lo K.Y., 1994, ApJ 433, 131

Zimmermann T., Ungerechts H., 1990, A\&A 238, 337

Ziurys L.M., Martin R.N., Pauls T.A., Wilson T.L., 1981 A\&A 104, 288 